\title{
Ergodicity Breaking in Thermal Biological Electron Transfer? Cytochrome C Revisited II
}

\author{
Zdenek Futera, ${ }^{a}$ Xiuyun Jiang, ${ }^{b}$ Jochen Blumberger ${ }^{b, *}$
}

March 30, 2020

* to whom correspondence should be addressed:

e-mail: j.blumberger@ucl.ac.uk (J.B.)

${ }^{a}$ Faculty of Science, University of South Bohemia, Branisovska 1760, 37005 Ceske Budejovice, Czech Republic.

${ }^{b}$ Department of Physics and Astronomy and Thomas Young Centre, University College London, London WC1E 6BT, UK.

\section{Supporting Information}




\section{Structural changes in Red/Ox MD simulations}

Both reduced and oxidized Cyt c simulations are based on reduced horse-heart cytochrome c crystal structure (pdb id IGIW), which was carefully equilibrated before $\sim 280$ ns production MD run. The computational setting is briefly described in the manuscript and all the details are stated in our previous work. [1] Here, we discuss the structural changes observed during the production MD (CHARMM27) runs with special focus on the charged protein residues as these are crucial for electrostatic potential and electric field analyzed in the main text.

The location of the charged amino acids in the protein structure is shown in Fig. S1(a) where the heme cofactor and the charged residues in the flexible part of the protein are labeled. There is a visible assymetry in location of negative (shown in red) and positive (blue) residues with respect to the heme cofactor, which is located in the central part of the protein. One side of the heme is exposed to solvent water (front side in Fig. S1(a)). The negatively charged amino acids are located mostly in the $\alpha$-helices where their charge is compensated by the simultaneous presence of the positively charged residues while the loops and coils on the other side of the protein are positively charged. This structurebound charge distribution determines the direction of the electric field acting on the heme cofactor, which points to the $\alpha$-helix region of the protein (see Fig. 1 in the main text).

Fluctuations of the field vector originate from fluctuations of the protein structure, in particular its charged regions, and fluctuations of the polar aquaous solution to which the heme cofactor is exposed. For the electric-field distribution, discussed in the main text, slower protein movements related to structural changes are crucial. From the calculated root-mean-square flucations (RMSF) of individual residues in reduced / oxidized Cyt c shown in Fig. S1(b) and S1(c), respectively, one can see that the loops and coils containing the positively charged residues are the most flexible parts of the protein compared to the rigid heme cofactor and well-preserved $\alpha$-helices, as expected.

Changes in this part of the protein can be seen in Fig. S2 where we aligned the protein structure of reduced and oxidized Cyt c from the same time of the production MD simulations. Indeed, while the $\alpha$-helices and the covalently-bound heme cofactors are practically unchanged, the flexible loops undergo significant conformational changes. To quantify the changes, we calculated the root-mean square deviation (RMSD) of protein atomic positions. The RMSD values of the rigid $\alpha$-helix regions are close to $2.1 \AA$, as expected, while the 3.5-4.0 $\AA$ values are obtained considering all backbone atoms including the flexible loops and coils. These values are consistent with work of Dinpajooh et al. [2]

For the electric field distributions, changes in position of the charged residues are crucial and therefore we tracked distances between Fe heme center and charged groups (CG carbon of aspartic acid and NZ nitrogen of lysine) of selected amino acids that are plotted in Fig. S3. Lys-27 and Lys-79 are closest to the Fe center $(\sim 12 \AA)$ and one expects that their positions will have the largest effect on the electric field distributions. However, the distance distributions of these two residues are very similar in both reduced and oxidized Cyt c MD simulations. Larger changes can be seen only in distributions of the most-distant Asp-50 and Lys-53, which are located on the short helix on the flexible loop (see Fig. S1(a)) 
and their distance from the Fe center fluctuates between 18 and $30 \AA$.

Nevertheless, the electric field is a superposition of all charged and dipolar aminoacid contributions which are inversely proportional to the distance and thus the effect of Asp-50 and Lys-53 movement is rather small because of their opposite charge and large distance to the Fe cation. In addition to the presented data, we also calculated the crosscorrelation functions between electric field vector and the position vector pointing from the Fe center to the charged groups. However, the correlation is rather small $(<0.18)$ for all charged amino acids and decays to zero within 20-40 ns. There is no particular charged amino acid strongly affecting the electric field distribution. The double-peak structure of the reduced Cyt c field distribution reported in [3] has probably originates from larger structural changes manifested by considerably different electric-field magnitude that we did not observe in our MD simulations. 

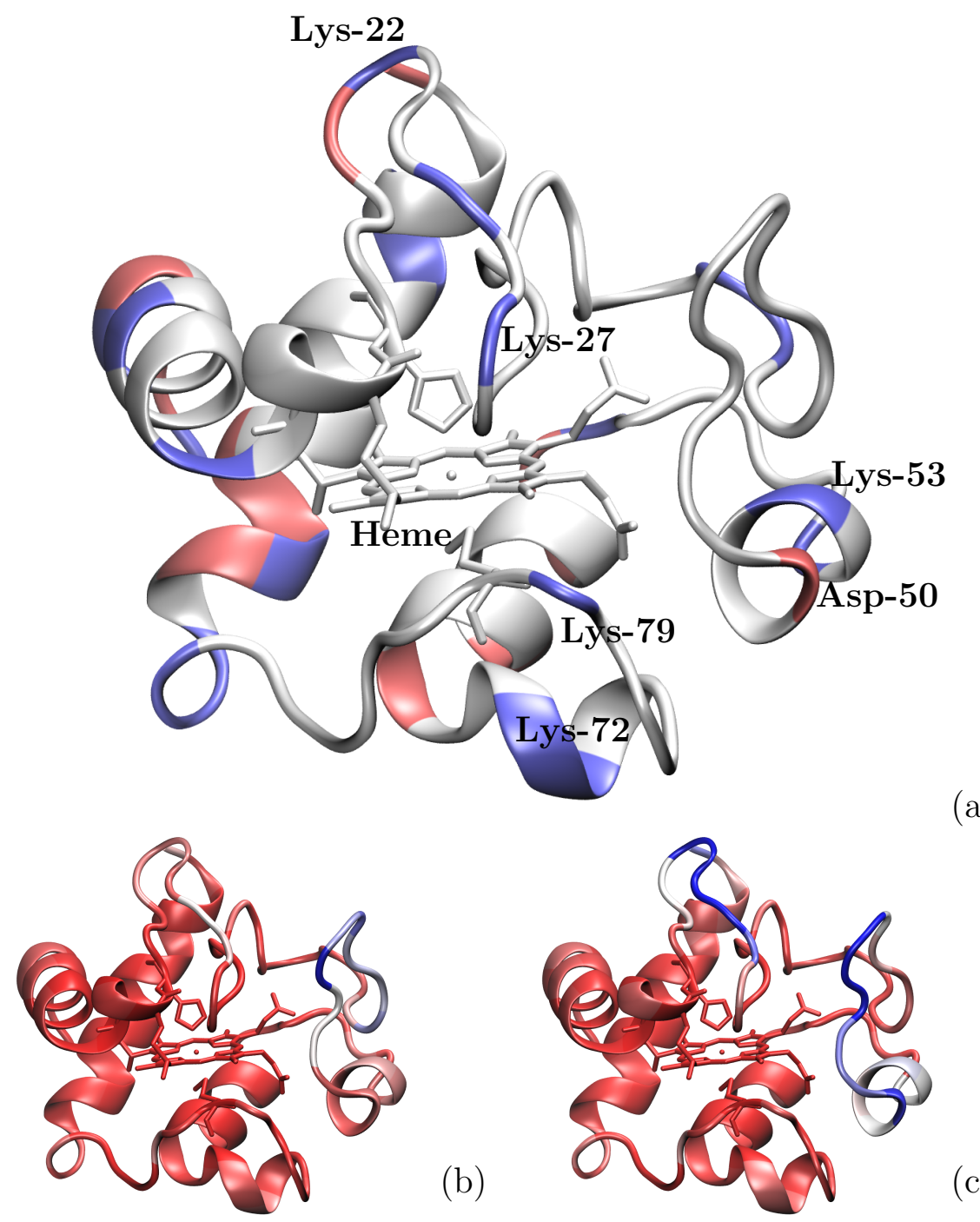

(b)

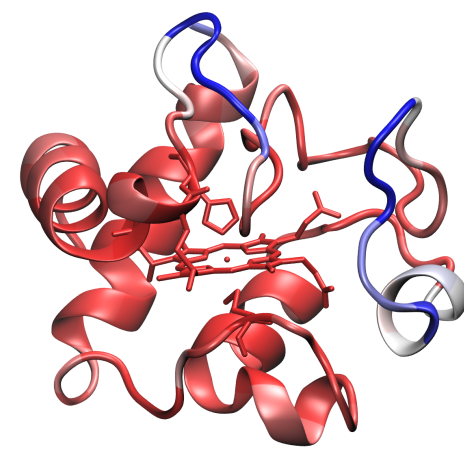

(a)

Figure S1: Crystal structure of Cyt c with (a) highlighted location of positively charged (blue) and negatively charged (red) amino acids. Root-mean-square fluctuations (RMSF) of Cyt c residues obtained from MD (CHARMM27) simulations of (b) reduced and (c) oxidized protein are shown by coloring the crystal structure where the red regions are the most rigid while the blue regions are the most flexible. 

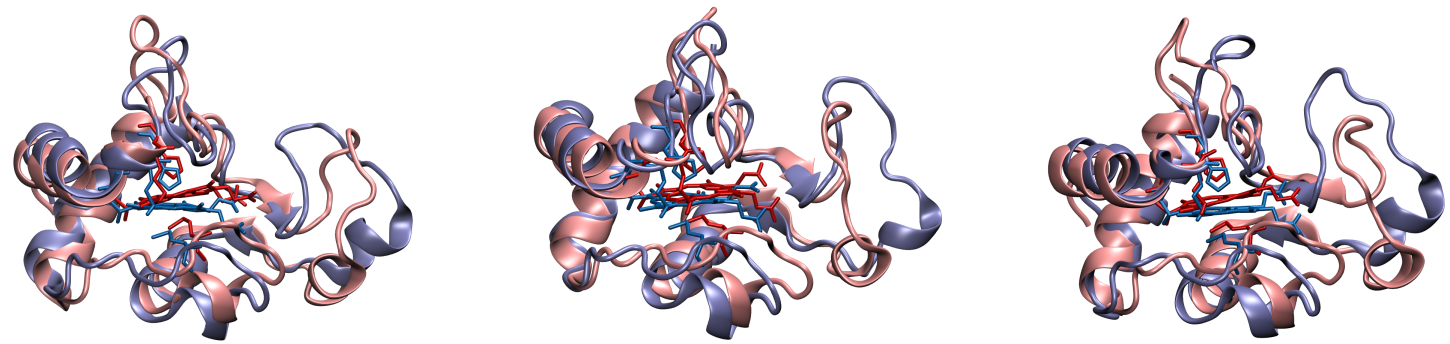

(a)

(b)

(c)
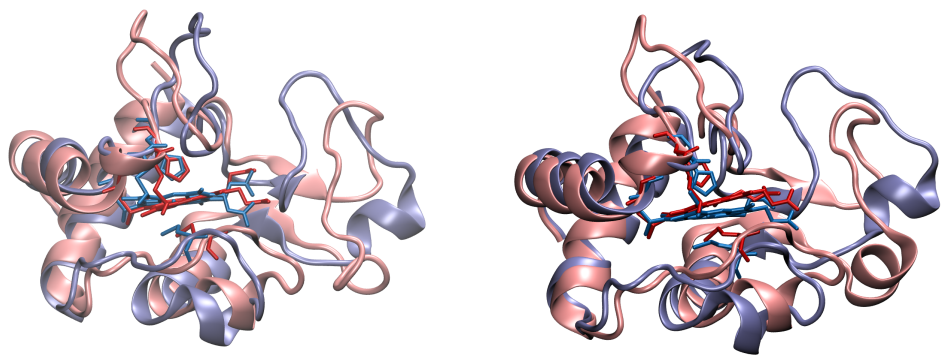

(d)

$(\mathrm{e})$

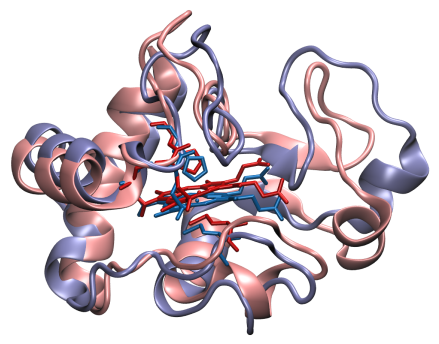

(f)

Figure S2: Snapthots of the Cyt c structures from MD simulations of reduced (red) oxidized (blue) proteins taken at times (a) $0 \mathrm{~ns}$, (b) $50 \mathrm{~ns}$, (c) $100 \mathrm{~ns}$, (d) $150 \mathrm{~ns}$, (e) $200 \mathrm{ns,} \mathrm{and}$ (f) $250 \mathrm{~ns}$ of the production run. For better comparison, the structures are aligned to minimize RMSD of their heavy atoms. 


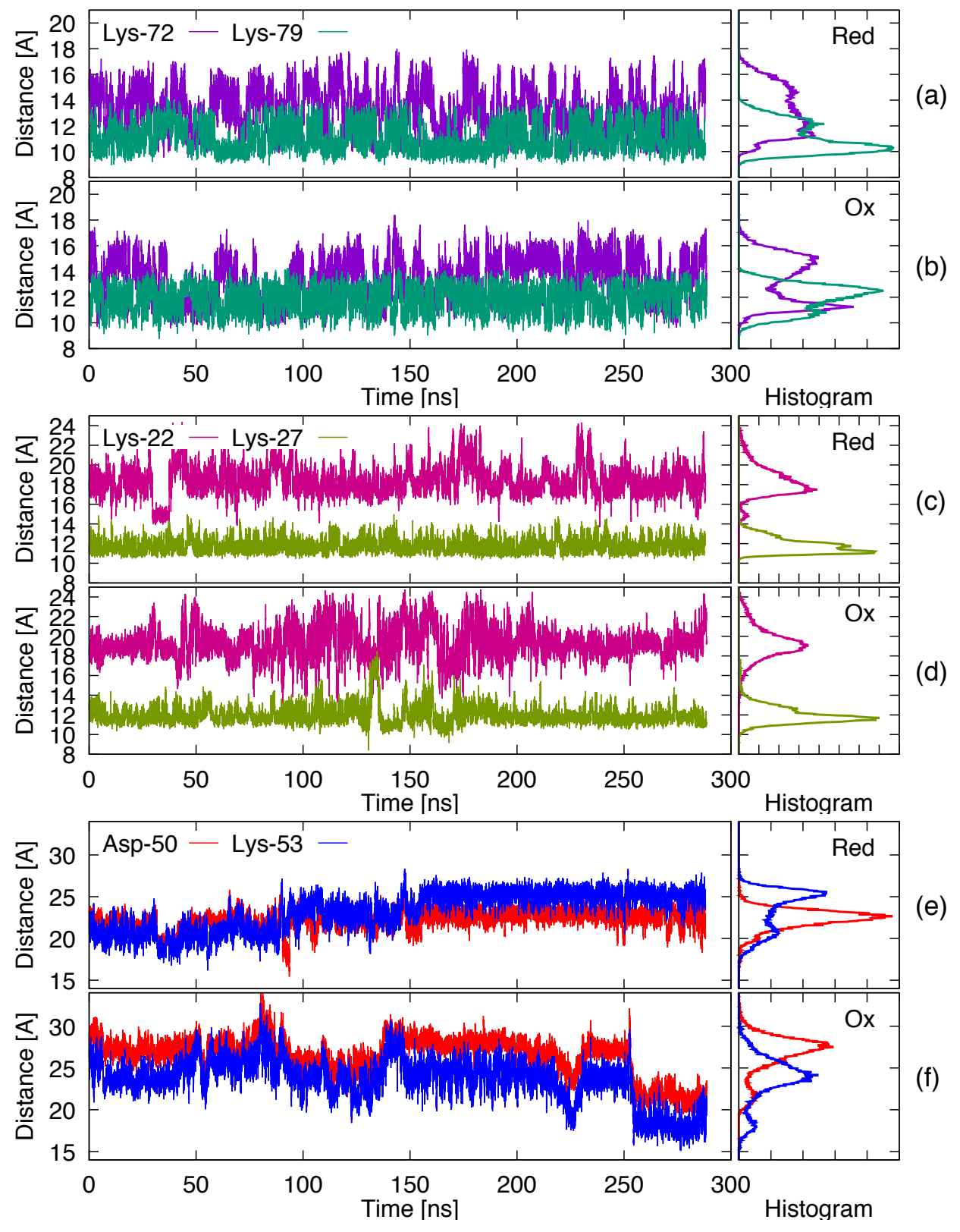

Figure S3: Distance tracks of selected charged amino acids from Fe center of the heme cofactor fo Cyt $\mathrm{c}$ and their histograms. Lys-72 and Lys-79 in reduced and oxidized protein MD are shown in panels (a) and (b), respectively; Lys-22 and Lys-27 in panels (c) and (d); and Asp-50 and Lys-53 in panels (e) and (f). 
Table S1: XYZ coordinates of reduced-state geometry in Gaussian 'standard orientation.

\begin{tabular}{|c|c|c|c|c|c|c|c|c|c|}
\hline $\mathrm{N}$ & Atom & $\mathrm{X}$ & $\mathrm{Y}$ & $\mathrm{Z}$ & $\mathrm{N}$ & Atom & $\mathrm{X}$ & $\mathrm{Y}$ & $\mathrm{Z}$ \\
\hline 1 & $\mathrm{~N}$ & -1.480726 & 7.296548 & 0.049539 & 69 & $\mathrm{O}$ & 2.255162 & -6.910552 & -5.228825 \\
\hline 2 & $\mathrm{C}$ & -0.878291 & 6.026047 & -0.339687 & 70 & $\mathrm{O}$ & 2.720797 & -4.953676 & -6.175498 \\
\hline 3 & $\mathrm{C}$ & 0.644708 & 6.171706 & -0.326545 & 71 & $\mathrm{~N}$ & -1.847976 & 0.873287 & -1.241374 \\
\hline 4 & $\mathrm{O}$ & 1.335929 & 5.304635 & 0.197848 & 72 & $\mathrm{C}$ & -2.233634 & 0.857422 & -2.544998 \\
\hline 5 & $\mathrm{C}$ & -1.318927 & 5.593874 & -1.718224 & 73 & $\mathrm{C}$ & -2.889485 & 2.121454 & -2.794366 \\
\hline 6 & $\mathrm{~S}$ & -3.103813 & 5.562919 & -2.092288 & 74 & $\mathrm{C}$ & -2.972548 & 2.807109 & -1.595877 \\
\hline 7 & $\mathrm{H}$ & -1.332422 & 8.088212 & -0.566519 & 75 & $\mathrm{C}$ & -2.256258 & 2.024346 & -0.624912 \\
\hline 8 & $\mathrm{H}$ & -1.146992 & 5.237104 & 0.359739 & 76 & $\mathrm{C}$ & -3.273104 & 2.630051 & -4.155331 \\
\hline 9 & $\mathrm{H}$ & -0.882292 & 6.305246 & -2.415051 & 77 & $\mathrm{C}$ & -3.747424 & 4.079244 & -1.280236 \\
\hline 10 & $\mathrm{H}$ & -0.894849 & 4.612114 & -1.924266 & 78 & $\mathrm{C}$ & -5.246720 & 3.922651 & -1.532865 \\
\hline 11 & $\mathrm{~N}$ & 3.197018 & 5.064796 & 2.312627 & 79 & $\mathrm{~N}$ & -0.711269 & 0.507207 & 1.415990 \\
\hline 12 & $\mathrm{C}$ & 3.640578 & 3.686083 & 2.253101 & 80 & $\mathrm{C}$ & -1.283499 & 1.714917 & 1.588149 \\
\hline 13 & $\mathrm{C}$ & 4.228790 & 3.331600 & 0.872588 & 81 & $\mathrm{C}$ & -0.950113 & 2.193180 & 2.903330 \\
\hline 14 & $\mathrm{O}$ & 5.040361 & 2.414242 & 0.795524 & 82 & $\mathrm{C}$ & -0.068423 & 1.287948 & 3.445941 \\
\hline 15 & $\mathrm{C}$ & 2.484931 & 2.783125 & 2.610452 & 83 & $\mathrm{C}$ & -0.023983 & 0.163881 & 2.539728 \\
\hline 16 & $\mathrm{~S}$ & 1.890655 & 3.052103 & 4.315435 & 84 & $\mathrm{C}$ & -1.397275 & 3.507659 & 3.489739 \\
\hline 17 & $\mathrm{H}$ & 2.321089 & 5.270144 & 1.853656 & 85 & $\mathrm{C}$ & 0.911765 & 1.538510 & 4.576053 \\
\hline 18 & $\mathrm{H}$ & 4.421795 & 3.514207 & 2.995871 & 86 & $\mathrm{C}$ & 0.304829 & 1.458769 & 5.977065 \\
\hline 19 & $\mathrm{H}$ & 1.665550 & 2.935057 & 1.906934 & 87 & $\mathrm{~N}$ & 0.349023 & -2.124827 & 0.617230 \\
\hline 20 & $\mathrm{H}$ & 2.827769 & 1.754931 & 2.524773 & 88 & C & 0.814433 & -2.043603 & 1.891841 \\
\hline 21 & $\mathrm{~N}$ & 3.834160 & 4.016270 & -0.215932 & 89 & $\mathrm{C}$ & 1.524278 & -3.261754 & 2.191083 \\
\hline 22 & $\mathrm{C}$ & 4.112499 & 3.603569 & -1.595631 & 90 & $\mathrm{C}$ & 1.474225 & -4.051266 & 1.066996 \\
\hline 23 & $\mathrm{C}$ & 4.624964 & 4.749303 & -2.463771 & 91 & $\mathrm{C}$ & 0.716815 & -3.319939 & 0.065483 \\
\hline 24 & $\mathrm{O}$ & 3.870012 & 5.306555 & -3.256350 & 92 & $\mathrm{C}$ & 2.236742 & -3.580242 & 3.484548 \\
\hline 25 & $\mathrm{C}$ & 2.812776 & 3.114993 & -2.236593 & 93 & $\mathrm{C}$ & 2.133033 & -5.408971 & 0.921812 \\
\hline 26 & C & 2.297354 & 1.837629 & -1.674240 & 94 & $\mathrm{C}$ & 1.156766 & -6.538392 & 0.577496 \\
\hline 27 & $\mathrm{~N}$ & 2.704813 & 0.594484 & -2.080701 & 95 & $\mathrm{C}$ & 0.832224 & -7.507660 & 1.715609 \\
\hline 28 & C & 1.116771 & 1.707571 & -0.999094 & 96 & $\mathrm{O}$ & 1.715955 & -7.863190 & 2.530267 \\
\hline 29 & $\mathrm{C}$ & 1.786173 & -0.272645 & -1.643972 & 97 & $\mathrm{O}$ & -0.338519 & -7.933979 & 1.718578 \\
\hline 30 & $\mathrm{~N}$ & 0.821597 & 0.337222 & -0.949872 & 98 & $\mathrm{H}$ & 0.825577 & -4.739357 & -1.504438 \\
\hline 31 & $\mathrm{H}$ & 3.204690 & 4.801772 & -0.090155 & 99 & $\mathrm{H}$ & -2.385763 & 0.038732 & -4.508760 \\
\hline 32 & $\mathrm{H}$ & 4.845769 & 2.795274 & -1.629392 & 100 & $\mathrm{H}$ & -2.404355 & 3.378849 & 1.037008 \\
\hline 33 & $\mathrm{H}$ & 2.044804 & 3.880712 & -2.116710 & 101 & $\mathrm{H}$ & 1.103953 & -1.122139 & 3.770590 \\
\hline 34 & $\mathrm{H}$ & 2.973507 & 2.947930 & -3.302828 & 102 & $\mathrm{H}$ & -2.405107 & -3.307831 & -5.698811 \\
\hline 35 & $\mathrm{H}$ & 3.443357 & 0.386142 & -2.746541 & 103 & $\mathrm{H}$ & -2.098364 & -1.589961 & -5.979172 \\
\hline 36 & $\mathrm{H}$ & 0.478946 & 2.550162 & -0.746965 & 104 & $\mathrm{H}$ & -0.831249 & -2.772182 & -6.303624 \\
\hline 37 & $\mathrm{H}$ & 1.737874 & -1.301971 & -1.934785 & 105 & $\mathrm{H}$ & -0.384066 & -5.534274 & -3.791797 \\
\hline 38 & $\mathrm{~N}$ & -1.206979 & -4.609487 & 4.488275 & 106 & $\mathrm{H}$ & -0.068912 & -4.651090 & -5.286473 \\
\hline 39 & $\mathrm{C}$ & -1.763710 & -3.323105 & 4.130087 & 107 & $\mathrm{H}$ & 2.141909 & -3.971240 & -3.960951 \\
\hline 40 & $\mathrm{C}$ & -2.807479 & -2.914696 & 5.163629 & 108 & $\mathrm{H}$ & 1.860440 & -5.554239 & -3.188005 \\
\hline 41 & $\mathrm{O}$ & -3.697453 & -3.683504 & 5.523465 & 109 & $\mathrm{H}$ & -4.020050 & 1.987448 & -4.619759 \\
\hline 42 & $\mathrm{C}$ & -2.371416 & -3.418444 & 2.739841 & 110 & $\mathrm{H}$ & -3.660065 & 3.638057 & -4.076698 \\
\hline 43 & $\mathrm{C}$ & -2.861768 & -2.096885 & 2.151829 & 111 & $\mathrm{H}$ & -2.374944 & 2.647976 & -4.768437 \\
\hline 44 & $\mathrm{~S}$ & -2.538833 & -1.975329 & 0.374467 & 112 & $\mathrm{H}$ & -3.682119 & 4.329915 & -0.229495 \\
\hline 45 & $\mathrm{C}$ & -4.139217 & -1.490258 & -0.323588 & 113 & $\mathrm{H}$ & -5.630683 & 3.124404 & -0.897394 \\
\hline 46 & $\mathrm{H}$ & -1.517812 & -5.402136 & 3.936200 & 114 & $\mathrm{H}$ & -5.758637 & 4.854896 & -1.299100 \\
\hline 47 & $\mathrm{H}$ & -0.952801 & -2.599281 & 4.111893 & 115 & $\mathrm{H}$ & -5.432970 & 3.656578 & -2.566663 \\
\hline 48 & $\mathrm{H}$ & -1.637428 & -3.865208 & 2.068377 & 116 & $\mathrm{H}$ & -2.486508 & 3.546650 & 3.479061 \\
\hline 49 & $\mathrm{H}$ & -3.237510 & -4.064116 & 2.824921 & 117 & $\mathrm{H}$ & -1.048009 & 3.624208 & 4.511318 \\
\hline 50 & $\mathrm{H}$ & -3.931973 & -2.023068 & 2.342634 & 118 & $\mathrm{H}$ & -0.998815 & 4.323509 & 2.887343 \\
\hline 51 & $\mathrm{H}$ & -2.386502 & -1.246145 & 2.633207 & 119 & $\mathrm{H}$ & 1.682286 & 0.778386 & 4.547676 \\
\hline 52 & $\mathrm{H}$ & -4.915189 & -2.199304 & -0.023451 & 110 & $\mathrm{H}$ & -0.040504 & 0.439131 & 6.144519 \\
\hline 53 & $\mathrm{H}$ & -4.386566 & -0.485482 & 0.021798 & 121 & $\mathrm{H}$ & 1.069509 & 1.699822 & 6.714636 \\
\hline 54 & $\mathrm{H}$ & -4.073021 & -1.499833 & -1.410587 & 122 & $\mathrm{H}$ & -0.529431 & 2.148904 & 6.083415 \\
\hline 55 & $\mathrm{Fe}$ & -0.793903 & -0.571543 & -0.375457 & 123 & $\mathrm{H}$ & 1.705540 & -3.155676 & 4.334967 \\
\hline 56 & $\mathrm{C}$ & 0.453192 & -3.769592 & -1.230921 & 124 & $\mathrm{H}$ & 2.295105 & -4.650634 & 3.637315 \\
\hline 57 & $\mathrm{C}$ & -2.016525 & -0.146640 & -3.514719 & 125 & $\mathrm{H}$ & 3.245062 & -3.174076 & 3.447425 \\
\hline 58 & $\mathrm{C}$ & -2.040351 & 2.429780 & 0.691086 & 126 & $\mathrm{H}$ & 2.893523 & -5.348147 & 0.144071 \\
\hline 59 & $\mathrm{C}$ & 0.675784 & -1.000469 & 2.793046 & 127 & $\mathrm{H}$ & 2.668157 & -5.681868 & 1.826086 \\
\hline 60 & $\mathrm{~N}$ & -0.826045 & -1.778757 & -2.065611 & 128 & $\mathrm{H}$ & 0.213992 & -6.097140 & 0.255498 \\
\hline 61 & $\mathrm{C}$ & -0.235305 & -3.018261 & -2.196159 & 129 & $\mathrm{H}$ & 1.558749 & -7.112226 & -0.256603 \\
\hline 62 & $\mathrm{C}$ & -0.395577 & -3.418788 & -3.576582 & 130 & $\mathrm{H}$ & -0.546347 & -4.745118 & 5.237430 \\
\hline 63 & $\mathrm{C}$ & -1.136514 & -2.438493 & -4.210582 & 131 & $\mathrm{H}$ & -2.750387 & -1.912322 & 5.585418 \\
\hline 64 & $\mathrm{C}$ & -1.361920 & -1.369435 & -3.254220 & 132 & $\mathrm{H}$ & 5.661457 & 5.072670 & -2.379880 \\
\hline 65 & $\mathrm{C}$ & -1.651439 & -2.521146 & -5.634306 & 133 & $\mathrm{H}$ & 1.115312 & 7.042240 & -0.781106 \\
\hline 66 & $\mathrm{C}$ & 0.143374 & -4.686343 & -4.219397 & 134 & $\mathrm{H}$ & -1.605809 & 7.624830 & 0.994340 \\
\hline 67 & $\mathrm{C}$ & 1.651758 & -4.934298 & -4.059300 & 135 & $\mathrm{H}$ & 3.707703 & 5.800007 & 2.776017 \\
\hline 68 & $\mathrm{C}$ & 2.266321 & -5.658857 & -5.251233 & & & & & \\
\hline
\end{tabular}


Table S2: XYZ coordinates of oxidized-state geometry in Gaussian 'standard orientation.

\begin{tabular}{|c|c|c|c|c|c|c|c|c|c|}
\hline $\mathrm{N}$ & Atom & $\mathrm{X}$ & $\mathrm{Y}$ & $\mathrm{Z}$ & $\mathrm{N}$ & Atom & $\mathrm{X}$ & $\mathrm{Y}$ & $\mathrm{Z}$ \\
\hline 1 & $\mathrm{~N}$ & -1.946256 & 7.731850 & -0.435258 & 69 & $\mathrm{O}$ & 3.698285 & -6.634842 & -3.713767 \\
\hline 2 & $\mathrm{C}$ & -1.189881 & 6.565452 & 0.005630 & 70 & $\mathrm{O}$ & 1.808869 & -7.378305 & -4.622103 \\
\hline 3 & $\mathrm{C}$ & 0.311140 & 6.605056 & -0.296143 & 71 & $\mathrm{~N}$ & -1.489513 & 0.738477 & -1.788024 \\
\hline 4 & $\mathrm{O}$ & 0.971544 & 5.567336 & -0.314938 & 72 & $\mathrm{C}$ & -1.568040 & 0.685418 & -3.148685 \\
\hline 5 & $\mathrm{C}$ & -1.850282 & 5.316410 & -0.526311 & 73 & $\mathrm{C}$ & -2.155713 & 1.927105 & -3.581800 \\
\hline 6 & $\mathrm{~S}$ & -2.268612 & 5.333383 & -2.298776 & 74 & $\mathrm{C}$ & -2.597294 & 2.586545 & -2.452432 \\
\hline 7 & $\mathrm{H}$ & -1.633473 & 8.212848 & -1.273275 & 75 & $\mathrm{C}$ & -2.120728 & 1.839967 & -1.311062 \\
\hline 8 & $\mathrm{H}$ & -1.250585 & 6.520684 & 1.094426 & 76 & $\mathrm{C}$ & -2.242513 & 2.403151 & -5.007680 \\
\hline 9 & $\mathrm{H}$ & $\begin{array}{l}-1.202926 \\
\text { S }\end{array}$ & 4.469069 & -0.317463 & 77 & $\mathrm{C}$ & -3.373653 & 3.885114 & -2.387826 \\
\hline 10 & $\mathrm{H}$ & $\begin{array}{r}-2.782636 \\
\end{array}$ & 5.206785 & 0.022940 & 78 & $\mathrm{C}$ & -4.452080 & 4.061912 & $\begin{array}{l}-3.460873 \\
\end{array}$ \\
\hline 11 & $\mathrm{~N}$ & 3.078320 & 5.157394 & 2.802779 & 79 & $\mathrm{~N}$ & -0.872170 & 0.529506 & 1.068767 \\
\hline 12 & $\mathrm{C}$ & 3.485358 & 3.761153 & 2.784348 & 80 & $\mathrm{C}$ & -1.585396 & 1.670146 & 1.086598 \\
\hline 13 & $\mathrm{C}$ & 4.276000 & 3.403937 & 1.524898 & 81 & $\mathrm{C}$ & -1.438696 & 2.262118 & 2.400174 \\
\hline 14 & $\mathrm{O}$ & 5.082378 & 2.480823 & 1.591842 & 82 & $\mathrm{C}$ & -0.561670 & 1.466471 & 3.105594 \\
\hline 15 & $\mathrm{C}$ & 2.286713 & 2.845360 & 2.933331 & 83 & $\mathrm{C}$ & -0.313802 & 0.298285 & 2.289122 \\
\hline 16 & $\mathrm{~S}$ & 1.228147 & 3.227284 & 4.364744 & 84 & $\mathrm{C}$ & -1.955229 & 3.613521 & 2.835088 \\
\hline 17 & $\mathrm{H}$ & 2.217477 & 5.382986 & 2.326032 & 85 & $\mathrm{C}$ & 0.113649 & 1.780727 & 4.435236 \\
\hline 18 & $\mathrm{H}$ & 4.169511 & 3.553490 & 3.600044 & 86 & $\mathrm{C}$ & -0.851745 & 1.908142 & 5.619478 \\
\hline 19 & $\mathrm{H}$ & 1.679344 & 2.888764 & 2.028651 & 87 & $\mathrm{~N}$ & 0.244844 & $\begin{array}{r}-2.100139 \\
\end{array}$ & 0.592904 \\
\hline 20 & $\mathrm{H}$ & 2.666458 & 1.831304 & 3.054722 & 88 & C & 0.594161 & -1.941577 & 1.895955 \\
\hline 21 & $\mathrm{~N}$ & 4.052510 & 4.084401 & 0.393125 & 89 & $\mathrm{C}$ & 1.205710 & -3.175931 & 2.334744 \\
\hline 22 & C & 4.424565 & 3.576439 & -0.928478 & 90 & $\mathrm{C}$ & 1.144158 & -4.055346 & 1.283200 \\
\hline 23 & $\mathrm{C}$ & 5.136442 & 4.609651 & -1.800623 & 91 & $\mathrm{C}$ & 0.556404 & -3.349663 & 0.181755 \\
\hline 24 & $\mathrm{O}$ & 4.950141 & 5.816870 & -1.676484 & 92 & $\mathrm{C}$ & 1.772540 & -3.476275 & 3.696943 \\
\hline 25 & $\mathrm{C}$ & 3.172375 & 3.104738 & -1.677282 & 93 & $\mathrm{C}$ & 1.492913 & -5.522780 & 1.324263 \\
\hline 26 & C & 2.645880 & 1.756651 & -1.269967 & 94 & $\mathrm{C}$ & 0.413379 & -6.324821 & 2.048188 \\
\hline 27 & $\mathrm{~N}$ & 3.111453 & 0.548425 & -1.740326 & 95 & $\mathrm{C}$ & 0.980038 & -7.534463 & 2.761676 \\
\hline 28 & C & 1.397511 & 1.540378 & -0.757544 & 96 & $\mathrm{O}$ & 0.498753 & -8.651828 & 2.490232 \\
\hline 29 & $\mathrm{C}$ & 2.156144 & -0.370601 & -1.521214 & 97 & $\mathrm{O}$ & 1.876975 & -7.337495 & 3.603592 \\
\hline 30 & $\mathrm{~N}$ & 1.092309 & 0.187971 & -0.921246 & 98 & $\mathrm{H}$ & 0.687183 & -4.920268 & -1.193381 \\
\hline 31 & $\mathrm{H}$ & 3.611255 & 4.999279 & 0.452176 & 99 & $\mathrm{H}$ & -1.338956 & -0.245773 & -5.063055 \\
\hline 32 & $\mathrm{H}$ & 5.098255 & 2.723590 & -0.827561 & 100 & $\mathrm{H}$ & -2.881632 & 3.091869 & 0.234239 \\
\hline 33 & $\mathrm{H}$ & 2.385128 & 3.846625 & -1.551743 & 101 & $\mathrm{H}$ & 0.690920 & -0.905075 & 3.727857 \\
\hline 34 & $\mathrm{H}$ & 3.392103 & 3.043829 & -2.743860 & 102 & $\mathrm{H}$ & -0.431760 & -1.971065 & -6.327372 \\
\hline 35 & $\mathrm{H}$ & 3.957544 & 0.405079 & -2.282798 & 103 & $\mathrm{H}$ & -0.018986 & -3.685116 & -6.207385 \\
\hline 36 & $\mathrm{H}$ & 0.709570 & 2.308634 & -0.432554 & 104 & $\mathrm{H}$ & -1.705811 & -3.177011 & -6.005684 \\
\hline 37 & $\mathrm{H}$ & 2.209522 & -1.394841 & -1.869546 & 105 & $\mathrm{H}$ & 0.359879 & -5.810421 & -3.024704 \\
\hline 38 & $\mathrm{~N}$ & -2.110729 & -4.099984 & 4.502166 & 106 & $\mathrm{H}$ & -0.067737 & -5.476466 & -4.626296 \\
\hline 39 & $\mathrm{C}$ & -2.650605 & -2.900751 & 3.890350 & 107 & $\mathrm{H}$ & 2.120046 & -4.617068 & -5.192420 \\
\hline 40 & $\mathrm{C}$ & -4.042306 & -2.558252 & 4.443139 & 108 & $\mathrm{H}$ & 2.544645 & -4.463284 & -3.476661 \\
\hline 41 & $\mathrm{O}$ & -5.055019 & -2.821169 & 3.796471 & 109 & $\mathrm{H}$ & -2.042776 & 3.471763 & -5.038744 \\
\hline 42 & $\mathrm{C}$ & -2.663880 & -3.137066 & 2.374199 & 110 & $\mathrm{H}$ & -1.489315 & 1.919050 & -5.626927 \\
\hline 43 & $\mathrm{C}$ & -3.212464 & -1.954196 & 1.577653 & 111 & $\mathrm{H}$ & -3.232841 & 2.183717 & -5.404300 \\
\hline 44 & $\mathrm{~S}$ & -2.605535 & -1.818460 & -0.120348 & 112 & $\mathrm{H}$ & -3.949324 & 3.889950 & -1.469740 \\
\hline 45 & $\mathrm{C}$ & -4.003160 & -1.013141 & -0.943031 & 113 & $\mathrm{H}$ & -5.190324 & 3.258202 & -3.403223 \\
\hline 46 & $\mathrm{H}$ & -2.029100 & -4.909881 & 3.913046 & 114 & $\mathrm{H}$ & -4.932157 & 5.017581 & -3.272137 \\
\hline 47 & $\mathrm{H}$ & -1.979729 & -2.065102 & 4.096063 & 115 & $\mathrm{H}$ & -4.042907 & 4.086851 & -4.460582 \\
\hline 48 & $\mathrm{H}$ & -1.651280 & -3.351603 & 2.049301 & 116 & $\mathrm{H}$ & -1.157463 & 4.347530 & 2.721379 \\
\hline 49 & $\mathrm{H}$ & -3.276764 & -4.006355 & 2.135711 & 117 & $\mathrm{H}$ & -2.810380 & 3.931718 & 2.244323 \\
\hline 50 & $\mathrm{H}$ & -4.293593 & -2.075293 & 1.522761 & 118 & $\mathrm{H}$ & -2.269969 & 3.580356 & 3.873157 \\
\hline 51 & $\mathrm{H}$ & -2.991958 & -1.014630 & 2.083502 & 119 & $\mathrm{H}$ & 0.785822 & 0.966797 & 4.683299 \\
\hline 52 & $\mathrm{H}$ & -4.911474 & -1.589667 & -0.773506 & 110 & $\mathrm{H}$ & -1.668509 & 1.192918 & 5.516022 \\
\hline 53 & $\mathrm{H}$ & -4.129570 & -0.006331 & -0.551072 & 121 & $\mathrm{H}$ & -0.312078 & 1.705811 & 6.544202 \\
\hline 54 & $\mathrm{H}$ & -3.809954 & -0.956041 & -2.013143 & 122 & $\mathrm{H}$ & -1.251837 & 2.918157 & 5.677760 \\
\hline 55 & $\mathrm{Fe}$ & -0.651528 & -0.630120 & -0.628372 & 123 & $\mathrm{H}$ & 2.758959 & -3.036994 & 3.783599 \\
\hline 56 & $\mathrm{C}$ & 0.371910 & -3.907216 & -1.053918 & 124 & $\mathrm{H}$ & 1.128996 & -3.070879 & 4.477462 \\
\hline 57 & $\mathrm{C}$ & -1.186032 & -0.354857 & -4.003323 & 125 & $\mathrm{H}$ & 1.878323 & -4.552449 & 3.818422 \\
\hline 58 & $\mathrm{C}$ & -2.284515 & 2.231540 & 0.024797 & 126 & $\mathrm{H}$ & 1.645807 & -5.960351 & 0.342559 \\
\hline 59 & $\mathrm{C}$ & 0.388089 & -0.831007 & 2.701944 & 127 & $\mathrm{H}$ & 2.446700 & -5.618966 & 1.816538 \\
\hline 60 & $\mathrm{~N}$ & -0.511675 & -1.922955 & -2.222297 & 128 & $\mathrm{H}$ & -0.058539 & -5.699768 & 2.805397 \\
\hline 61 & $\mathrm{C}$ & -0.078074 & -3.201397 & -2.146605 & 129 & $\mathrm{H}$ & -0.336279 & -6.633543 & 1.318902 \\
\hline 62 & $\mathrm{C}$ & -0.050608 & -3.741601 & -3.470378 & 130 & $\mathrm{H}$ & -1.823647 & -4.147744 & 5.467239 \\
\hline 63 & $\mathrm{C}$ & -0.470792 & -2.753905 & -4.329413 & 131 & $\mathrm{H}$ & -4.127417 & -2.078328 & 5.416972 \\
\hline 64 & $\mathrm{C}$ & -0.743307 & -1.584712 & -3.521913 & 132 & $\mathrm{H}$ & 3.647331 & 5.842055 & 3.275569 \\
\hline 65 & $\mathrm{C}$ & -0.668408 & -2.902067 & -5.816150 & 133 & $\mathrm{H}$ & 5.830633 & 4.240477 & -2.554103 \\
\hline 66 & $\mathrm{C}$ & 0.512662 & -5.088802 & -3.822574 & 134 & $\mathrm{H}$ & 0.793346 & 7.561019 & -0.494977 \\
\hline 67 & $\mathrm{C}$ & 1.994791 & -5.059878 & -4.204480 & 135 & $\mathrm{H}$ & -2.756501 & 8.064168 & 0.063873 \\
\hline 68 & $\mathrm{C}$ & 2.562020 & -6.470181 & -4.203444 & & & & & \\
\hline
\end{tabular}


Table S3: Energies $E_{i}[\mathrm{eV}]$ and dipole moments $\boldsymbol{\mu}_{i i} X, Y, Z$ components [D] obtained by $\mathrm{ZINDO} / \mathrm{S}$ for reduced state on reduced-state geometry.

\begin{tabular}{|c|c|c|c|c|c|c|c|c|c|}
\hline $\mathrm{i}$ & $E_{i}$ & $\mu_{i i}^{x}$ & $\mu_{i i}^{y}$ & $\mu_{i i}^{z}$ & $\mathrm{i}$ & $E_{i}$ & $\mu_{i i}^{x}$ & $\mu_{i i}^{y}$ & $\mu_{i i}^{z}$ \\
\hline 0 & 0.000 & -9.2896 & 64.4154 & 11.8794 & 51 & 4.131 & -17.6206 & 23.3051 & 0.5821 \\
\hline 1 & 0.863 & -8.9808 & 64.4811 & 11.6871 & 52 & 4.142 & -15.4250 & 36.2932 & 12.1771 \\
\hline 2 & 0.913 & -9.0902 & 64.5458 & 11.6457 & 53 & 4.177 & -26.9368 & 50.6093 & 10.1551 \\
\hline 3 & 1.038 & -9.2154 & 64.4905 & 11.7811 & 54 & 4.183 & -13.1175 & 17.3003 & 18.2331 \\
\hline 4 & 1.756 & -9.1383 & 63.5624 & 11.2376 & 55 & 4.228 & 1.6438 & 75.4007 & 10.1907 \\
\hline 5 & 1.795 & -9.5330 & 65.1874 & 12.2579 & 56 & 4.275 & -15.0405 & 37.6546 & 11.3652 \\
\hline 6 & 2.121 & -9.1255 & 64.4787 & 11.6804 & 57 & 4.308 & -9.0507 & 66.8929 & 14.9575 \\
\hline 7 & 2.185 & -9.1594 & 64.5323 & 11.7575 & 58 & 4.331 & -18.9563 & 60.3641 & 15.7367 \\
\hline 8 & 2.222 & -9.0764 & 64.5351 & 11.6700 & 59 & 4.353 & -9.0488 & 62.8678 & 11.3408 \\
\hline 9 & 2.723 & -8.2122 & 63.5890 & 12.3146 & 60 & 4.358 & -28.1138 & 15.1776 & 29.4451 \\
\hline 10 & 2.731 & -16.1008 & 38.7328 & 11.7866 & 61 & 4.407 & -11.4943 & 63.5151 & 11.7573 \\
\hline 11 & 2.776 & -9.0311 & 63.6722 & 9.9155 & 62 & 4.417 & -11.2861 & 10.6114 & 21.6305 \\
\hline 12 & 2.826 & -8.6008 & 63.9841 & 11.3676 & 63 & 4.429 & -10.2364 & 66.3094 & 10.8840 \\
\hline 13 & 2.963 & -8.4549 & 62.5784 & 12.0365 & 64 & 4.448 & -9.4931 & 19.5636 & -5.3254 \\
\hline 14 & 2.994 & -8.1798 & 61.1260 & 12.3065 & 65 & 4.464 & -11.9813 & 10.1127 & 21.5680 \\
\hline 15 & 3.121 & -8.9964 & 63.4479 & 10.5996 & 66 & 4.469 & -12.9331 & 20.2503 & -3.2550 \\
\hline 16 & 3.133 & -10.4323 & 64.7758 & 11.0588 & 67 & 4.473 & -10.5963 & 65.0798 & 11.6796 \\
\hline 17 & 3.164 & -10.1042 & 63.4298 & 12.3560 & 68 & 4.482 & -10.6247 & 19.7895 & -4.9854 \\
\hline 18 & 3.203 & -9.0740 & 60.6076 & 12.4800 & 69 & 4.502 & -11.9886 & 20.0926 & -4.1525 \\
\hline 19 & 3.208 & -6.0001 & 50.1552 & 14.5950 & 70 & 4.506 & -10.2762 & 64.4075 & 12.0586 \\
\hline 20 & 3.252 & -31.5201 & 42.2693 & 20.7752 & 71 & 4.532 & -19.9646 & 55.9511 & 14.6186 \\
\hline 21 & 3.257 & -15.6019 & 41.3223 & 11.9909 & 72 & 4.540 & -27.2857 & 45.6266 & 18.9258 \\
\hline 22 & 3.262 & -8.2354 & 63.7789 & 12.4494 & 73 & 4.563 & -7.3609 & 63.2237 & 13.4837 \\
\hline 23 & 3.312 & -9.1046 & 64.1512 & 10.2979 & 74 & 4.580 & -8.1833 & 63.9577 & 9.8706 \\
\hline 24 & 3.323 & -2.6545 & 50.9192 & 2.3475 & 75 & 4.587 & -26.7658 & 13.6707 & 30.6844 \\
\hline 25 & 3.343 & -19.5697 & 60.0706 & 15.7825 & 76 & 4.591 & -9.0305 & 63.9276 & 10.6497 \\
\hline 26 & 3.421 & -5.8446 & 40.2674 & 17.6760 & 77 & 4.620 & 3.3284 & 61.4385 & -4.7540 \\
\hline 27 & 3.453 & -5.0620 & 50.9786 & 13.1435 & 78 & 4.623 & -8.9508 & 63.2536 & 9.8911 \\
\hline 28 & 3.465 & -0.5689 & 47.6937 & -0.5914 & 79 & 4.639 & -27.4141 & 13.1360 & 30.6744 \\
\hline 29 & 3.547 & -5.8182 & 51.2783 & 10.4574 & 80 & 4.645 & -28.9428 & 48.5285 & 9.1842 \\
\hline 30 & 3.555 & -5.3634 & 50.2496 & 11.1710 & 81 & 4.657 & -8.2861 & 65.0323 & 10.8238 \\
\hline 31 & 3.568 & -8.1722 & 56.7701 & 14.2924 & 82 & 4.674 & 3.2191 & 61.9964 & -5.4698 \\
\hline 32 & 3.607 & -5.0341 & 35.9139 & 21.5415 & 83 & 4.706 & -8.3646 & 41.9839 & -2.8815 \\
\hline 33 & 3.677 & -19.5719 & 59.6119 & 15.8732 & 84 & 4.720 & -7.6232 & 63.8919 & 10.6320 \\
\hline 34 & 3.743 & -31.5032 & 42.1570 & 20.8715 & 85 & 4.734 & -31.2354 & 41.6779 & 20.2251 \\
\hline 35 & 3.747 & 3.2731 & 44.0096 & -6.6045 & 86 & 4.777 & -10.9985 & 47.4736 & 10.0549 \\
\hline 36 & 3.750 & -4.3111 & 36.4747 & 19.8873 & 87 & 4.782 & -12.7287 & 55.9814 & 11.8942 \\
\hline 37 & 3.771 & -7.9460 & 63.1373 & 12.2744 & 88 & 4.803 & -11.1398 & 52.0299 & 10.9153 \\
\hline 38 & 3.787 & 2.2627 & 44.6893 & -4.5855 & 89 & 4.805 & -10.8675 & 52.6091 & 10.8026 \\
\hline 39 & 3.790 & -4.4584 & 36.5605 & 18.2433 & 90 & 4.824 & -3.3627 & 62.6695 & 11.5582 \\
\hline 40 & 3.802 & -7.4618 & 62.6761 & 13.1237 & 91 & 4.854 & -3.3719 & 61.8363 & 10.8719 \\
\hline 41 & 3.874 & -6.2696 & 56.7622 & 13.5144 & 92 & 4.864 & -5.6382 & 60.4322 & 11.8238 \\
\hline 42 & 3.877 & 3.0774 & 44.6176 & -8.2363 & 93 & 4.872 & -22.6958 & 47.6468 & 17.3035 \\
\hline 43 & 3.917 & 3.2929 & 44.1285 & -9.3895 & 94 & 4.880 & -4.9483 & 61.7057 & 11.8876 \\
\hline 44 & 3.946 & -10.3305 & 64.3986 & 11.5619 & 95 & 4.910 & -15.5747 & 41.2315 & 14.8761 \\
\hline 45 & 3.979 & -17.3659 & 60.9781 & 14.6952 & 96 & 4.929 & 6.3837 & 46.9465 & -0.1148 \\
\hline 46 & 3.985 & -8.4825 & 61.1199 & 6.0806 & 97 & 4.930 & -6.7462 & 62.5640 & 9.4866 \\
\hline 47 & 4.018 & -17.3542 & 60.7778 & 14.7215 & 98 & 4.963 & 6.9720 & 47.1945 & 0.0882 \\
\hline 48 & 4.060 & -10.7519 & 65.4493 & 10.6525 & 99 & 4.967 & -7.2366 & 45.0603 & 15.7642 \\
\hline 49 & 4.104 & -7.6463 & 21.4281 & -4.9712 & 100 & 4.972 & -15.1051 & 37.6591 & 22.5982 \\
\hline 50 & 4.120 & -22.6306 & 53.6531 & 12.6061 & & & & & \\
\hline
\end{tabular}


Table S4: Energies $E_{i}[\mathrm{eV}]$ and dipole moments $\boldsymbol{\mu}_{i i} X, Y, Z$ components [D] obtained by ZINDO/S for oxidized state on reduced-state geometry.

\begin{tabular}{|c|c|c|c|c|c|c|c|c|c|}
\hline $\mathrm{i}$ & $E_{i}$ & $\mu_{i i}^{x}$ & $\mu_{i i}^{y}$ & $\mu_{i i}^{z}$ & $\overline{\mathrm{i}}$ & $E_{i}$ & $\mu_{i i}^{x}$ & $\mu_{i i}^{y}$ & $\mu_{i i}^{z}$ \\
\hline 0 & 0.000 & $\frac{w}{-13.0324}$ & 63.8643 & 10.4407 & 51 & 3.175 & -10.7077 & 58.1732 & 8.4700 \\
\hline 1 & 0.404 & -13.1849 & 63.7260 & 10.4278 & 52 & 3.205 & -6.1467 & 34.9427 & 24.4783 \\
\hline 2 & 0.459 & -13.2657 & 63.5337 & 10.4587 & 53 & 3.230 & -10.6520 & 39.2353 & 13.6047 \\
\hline 3 & 0.585 & -13.2711 & 63.4400 & 10.5283 & 54 & 3.234 & -12.1575 & 62.6353 & 11.0078 \\
\hline 4 & 0.888 & -12.7776 & 63.8541 & 10.2763 & 55 & 3.237 & -6.3026 & 34.5694 & 24.4018 \\
\hline 5 & 0.960 & -12.9177 & 63.8084 & 10.3015 & 56 & 3.261 & -1.6056 & 41.8861 & -15.2481 \\
\hline 6 & 0.983 & -12.9011 & 63.7468 & 10.2967 & 57 & 3.291 & -8.9607 & 42.7103 & 19.0519 \\
\hline 7 & 1.055 & -13.2917 & 63.5027 & 10.4783 & 58 & 3.293 & -1.8465 & 42.0058 & -15.1046 \\
\hline 8 & 1.116 & -13.1560 & 63.5789 & 10.4088 & 59 & 3.332 & -10.8868 & 52.1466 & 10.3589 \\
\hline 9 & 1.126 & -13.2203 & 63.7040 & 10.4645 & 60 & 3.362 & -11.4903 & 62.8059 & 12.0867 \\
\hline 10 & 1.386 & -10.0621 & 57.0836 & 9.3372 & 61 & 3.382 & -11.2264 & 58.3973 & 10.2785 \\
\hline 11 & 1.575 & -10.2427 & 43.9045 & 15.7018 & 62 & 3.388 & -11.4779 & 62.3948 & 10.8963 \\
\hline 12 & 1.604 & -6.6247 & 47.9914 & 2.4608 & 63 & 3.434 & -7.6676 & 53.3026 & 1.6319 \\
\hline 13 & 1.711 & -7.2377 & 50.7024 & 1.6896 & 64 & 3.455 & -6.7741 & 51.7269 & -0.3249 \\
\hline 14 & 1.857 & -7.4905 & 34.4611 & 20.1234 & 65 & 3.490 & -10.9519 & 53.2759 & 8.9164 \\
\hline 15 & 1.892 & -8.1265 & 33.7321 & 20.1481 & 66 & 3.497 & -3.3171 & 40.4125 & 3.4960 \\
\hline 16 & 1.898 & -12.4059 & 63.7294 & 12.3991 & 67 & 3.498 & -5.4183 & 43.7696 & 4.9646 \\
\hline 17 & 1.979 & 0.2225 & 42.1663 & -11.0272 & 68 & 3.533 & -10.0453 & 34.9304 & 15.9283 \\
\hline 18 & 2.022 & 0.0562 & 42.1149 & -11.1439 & 69 & 3.540 & 1.7278 & 41.7333 & -5.7731 \\
\hline 19 & 2.091 & -12.6019 & 63.8232 & 10.1382 & 70 & 3.557 & -9.7781 & 53.9647 & 12.3389 \\
\hline 20 & 2.218 & -11.4897 & 59.8887 & 9.5400 & 71 & 3.572 & -6.8604 & 39.4032 & 21.3232 \\
\hline 21 & 2.256 & -13.2251 & 63.6534 & 10.4524 & 72 & 3.586 & -11.5191 & 59.6562 & 11.2877 \\
\hline 22 & 2.277 & -12.7869 & 63.6747 & 10.1776 & 73 & 3.596 & -6.1964 & 34.8553 & 23.3556 \\
\hline 23 & 2.345 & -10.5532 & 56.8299 & 8.9386 & 74 & 3.614 & -22.5438 & 60.6233 & 13.8911 \\
\hline 24 & 2.519 & -11.2489 & 58.5065 & 9.5583 & 75 & 3.643 & -13.2920 & 61.7693 & 8.3393 \\
\hline 25 & 2.529 & -11.2824 & 50.1302 & 9.6227 & 76 & 3.652 & -12.7044 & 62.4069 & 9.3958 \\
\hline 26 & 2.554 & -12.1230 & 60.1052 & 10.4368 & 77 & 3.710 & -12.6950 & 63.5446 & 10.3098 \\
\hline 27 & 2.570 & -11.7623 & 50.9055 & 9.5282 & 78 & 3.757 & -11.6935 & 62.6194 & 10.8628 \\
\hline 28 & 2.644 & -3.7488 & 49.7884 & 2.6029 & 79 & 3.777 & -2.0208 & 43.0494 & -14.1178 \\
\hline 29 & 2.673 & -8.7990 & 56.7794 & 6.6489 & 80 & 3.801 & -11.0809 & 60.4895 & 10.7250 \\
\hline 30 & 2.702 & -14.2362 & 62.7432 & 11.2257 & 81 & 3.820 & -2.3746 & 43.3857 & -13.8302 \\
\hline 31 & 2.704 & -14.1670 & 64.6598 & 9.2507 & 82 & 3.836 & -14.3652 & 62.1349 & 11.4557 \\
\hline 32 & 2.777 & -12.3007 & 61.4154 & 7.3837 & 83 & 3.845 & -10.4699 & 59.8310 & 10.4531 \\
\hline 33 & 2.823 & -9.1932 & 33.4160 & 13.5386 & 84 & 3.916 & -11.7710 & 63.0040 & 9.2005 \\
\hline 34 & 2.824 & -11.9228 & 62.4626 & 11.1124 & 85 & 3.918 & -12.5930 & 61.9605 & 8.6365 \\
\hline 35 & 2.870 & -9.7697 & 32.9029 & 13.5450 & 86 & 3.933 & -7.9096 & 19.2701 & -8.8291 \\
\hline 36 & 2.884 & -10.3960 & 64.2551 & 8.8213 & 87 & 3.935 & -11.9835 & 54.1210 & 3.5454 \\
\hline 37 & 2.902 & -10.1358 & 54.9701 & 10.9907 & 88 & 3.936 & -11.5684 & 58.6103 & 5.8711 \\
\hline 38 & 2.912 & -3.4375 & 43.6118 & -10.9171 & 89 & 3.940 & -12.0912 & 53.0866 & 2.8148 \\
\hline 39 & 2.935 & -8.8906 & 42.6387 & 19.6586 & 90 & 3.943 & -13.3217 & 61.8915 & 11.7998 \\
\hline 40 & 2.962 & -11.6775 & 57.8270 & 6.1509 & 91 & 3.951 & -8.2874 & 20.1648 & -8.1892 \\
\hline 41 & 3.003 & -12.2043 & 64.0229 & 13.8860 & 92 & 3.955 & -13.9551 & 62.2657 & 9.0725 \\
\hline 42 & 3.015 & -11.4324 & 60.0299 & 9.3824 & 93 & 3.988 & -14.3543 & 64.9329 & 8.8885 \\
\hline 43 & 3.021 & -12.2091 & 61.1837 & 8.7911 & 94 & 4.012 & -18.5850 & 60.3008 & 12.6495 \\
\hline 44 & 3.030 & -10.9512 & 59.3020 & 9.0011 & 95 & 4.036 & -16.3343 & 11.3618 & 19.9625 \\
\hline 45 & 3.048 & 1.3789 & 43.0612 & -3.6970 & 96 & 4.041 & -16.5810 & 12.8475 & 19.6177 \\
\hline 46 & 3.076 & -3.5144 & 50.2479 & 1.4883 & 97 & 4.045 & -12.8225 & 61.5359 & 9.9602 \\
\hline 47 & 3.085 & -7.2605 & 55.0695 & 5.4148 & 98 & 4.074 & -8.2861 & 64.0915 & 9.1036 \\
\hline 48 & 3.132 & -13.9614 & 62.9045 & 11.0225 & 99 & 4.078 & -11.2072 & 61.8666 & 11.4998 \\
\hline 49 & 3.144 & 0.2004 & 43.6313 & -3.8574 & 100 & 4.088 & -11.6321 & 61.7419 & 9.8865 \\
\hline 50 & 3.144 & -13.7144 & 63.9925 & 9.1852 & & & & & \\
\hline
\end{tabular}


Table S5: Energies $E_{i}[\mathrm{eV}]$ and dipole moments $\boldsymbol{\mu}_{i i} X, Y, Z$ components [D] obtained by ZINDO/S for oxidized state on oxidized-state geometry.

\begin{tabular}{|c|c|c|c|c|c|c|c|c|c|}
\hline $\mathrm{i}$ & $E_{i}$ & $\mu_{i i}^{x}$ & $\mu_{i i}^{y}$ & $\mu_{i i}^{z}$ & $\mathrm{i}$ & $E_{i}$ & $\mu_{i i}^{x}$ & $\mu_{i i}^{y}$ & $\mu_{i i}^{z}$ \\
\hline 0 & 0.000 & -20.7563 & 66.1767 & 1.0402 & 51 & 3.208 & -20.8344 & 67.7277 & 1.2159 \\
\hline 1 & 0.378 & -20.8304 & 66.1825 & 1.0594 & 52 & 3.219 & -19.8007 & 64.6628 & 0.9375 \\
\hline 2 & 0.496 & -20.7559 & 66.2265 & 1.0890 & 53 & 3.222 & -19.0354 & 63.7586 & 3.6128 \\
\hline 3 & 0.595 & -21.0589 & 65.9166 & 1.1949 & 54 & 3.267 & -19.1499 & 63.1816 & 3.6555 \\
\hline 4 & 0.888 & -20.4858 & 66.3208 & 0.9155 & 55 & 3.282 & -22.5455 & 71.6931 & 4.2635 \\
\hline 5 & 0.921 & -20.6273 & 66.0853 & 0.9812 & 56 & 3.335 & -14.5631 & 40.2423 & 13.8780 \\
\hline 6 & 0.979 & -20.7960 & 66.0754 & 1.1517 & 57 & 3.369 & -19.2555 & 64.7559 & 1.7284 \\
\hline 7 & 1.047 & -20.7411 & 66.1262 & 0.9945 & 58 & 3.382 & -10.5759 & 46.6547 & -5.9676 \\
\hline 8 & 1.124 & -20.6815 & 66.3320 & 1.0503 & 59 & 3.384 & -9.6799 & 45.6551 & -6.8290 \\
\hline 9 & 1.184 & -20.9217 & 66.2107 & 1.0377 & 60 & 3.396 & -14.1946 & 42.7206 & 7.3833 \\
\hline 10 & 1.270 & -16.1673 & 50.0626 & -6.7585 & 61 & 3.403 & -8.3715 & 45.3727 & -8.4929 \\
\hline 11 & 1.455 & -13.8675 & 35.2774 & 8.5475 & 62 & 3.414 & -14.7115 & 41.0617 & 18.1409 \\
\hline 12 & 1.529 & -7.2964 & 38.0333 & -16.6135 & 63 & 3.436 & -17.2052 & 55.7030 & 3.2120 \\
\hline 13 & 1.734 & -12.9531 & 31.3107 & 12.1363 & 64 & 3.449 & -14.9363 & 40.3984 & 18.3566 \\
\hline 14 & 1.777 & -22.7185 & 71.3955 & 6.2966 & 65 & 3.460 & -17.8148 & 51.1114 & 9.3299 \\
\hline 15 & 1.791 & -13.2595 & 31.6279 & 11.9124 & 66 & 3.464 & -19.1734 & 59.6012 & 4.1338 \\
\hline 16 & 1.835 & -5.1460 & 34.3742 & -18.9371 & 67 & 3.509 & -17.0181 & 60.0317 & 2.4260 \\
\hline 17 & 1.836 & -5.3832 & 34.2877 & -18.7519 & 68 & 3.516 & -8.2495 & 45.3617 & -8.8256 \\
\hline 18 & 1.968 & -18.8014 & 59.5374 & -3.4171 & 69 & 3.525 & -9.0295 & 46.2241 & -7.9793 \\
\hline 19 & 2.057 & -17.9294 & 58.0488 & 1.0436 & 70 & 3.545 & -20.8070 & 67.9751 & 3.5443 \\
\hline 20 & 2.103 & -19.7174 & 63.6746 & 1.3978 & 71 & 3.583 & -18.9847 & 60.4093 & 2.8452 \\
\hline 21 & 2.212 & -19.1519 & 56.7454 & 3.6410 & 72 & 3.636 & -20.9924 & 67.5755 & 2.9292 \\
\hline 22 & 2.232 & -20.3598 & 63.9798 & 1.8539 & 73 & 3.649 & -13.1431 & 36.3637 & 16.5769 \\
\hline 23 & 2.274 & -20.5206 & 66.2378 & 0.9000 & 74 & 3.702 & -13.4885 & 36.6132 & 16.0435 \\
\hline 24 & 2.330 & -20.8436 & 67.9073 & 3.5284 & 75 & 3.721 & -8.0111 & 42.5090 & -12.3906 \\
\hline 25 & 2.435 & -22.1498 & 71.8405 & 4.8968 & 76 & 3.724 & -23.9575 & 60.0974 & 1.4278 \\
\hline 26 & 2.459 & -19.2380 & 59.8352 & 4.5386 & 77 & 3.730 & -9.4259 & 43.5945 & -11.4302 \\
\hline 27 & 2.491 & -19.6057 & 61.5366 & -2.7956 & 78 & 3.739 & -19.4583 & 64.9691 & 1.6521 \\
\hline 28 & 2.552 & -19.8649 & 63.2598 & -0.6379 & 79 & 3.759 & -23.5313 & 62.5190 & 3.1108 \\
\hline 29 & 2.582 & -19.8021 & 64.2193 & -1.3633 & 80 & 3.788 & -23.0175 & 73.1690 & 3.1658 \\
\hline 30 & 2.652 & -19.2545 & 61.3844 & 0.7114 & 81 & 3.821 & -20.4054 & 67.4803 & 2.4761 \\
\hline 31 & 2.683 & -22.0205 & 65.7659 & 0.2470 & 82 & 3.853 & -18.7844 & 50.0153 & -6.8003 \\
\hline 32 & 2.716 & -17.5538 & 48.5757 & 9.7745 & 83 & 3.856 & -18.2858 & 53.1408 & -5.8658 \\
\hline 33 & 2.752 & -17.6857 & 56.7831 & -4.2617 & 84 & 3.872 & -18.6403 & 63.5256 & -1.8257 \\
\hline 34 & 2.762 & -14.7927 & 56.8462 & -5.8603 & 85 & 3.890 & -19.5127 & 63.0023 & 0.1579 \\
\hline 35 & 2.773 & -17.3745 & 44.7460 & 12.0356 & 86 & 3.897 & -19.3977 & 65.4277 & -0.0096 \\
\hline 36 & 2.775 & -20.7908 & 68.0370 & 1.1964 & 87 & 3.936 & -19.2572 & 62.6586 & 3.8979 \\
\hline 37 & 2.786 & -19.5717 & 64.3932 & 0.9065 & 88 & 3.944 & -20.0727 & 64.0805 & 2.0636 \\
\hline 38 & 2.787 & -17.5725 & 61.4941 & -1.3898 & 89 & 3.948 & -19.4088 & 63.9467 & 0.9569 \\
\hline 39 & 2.866 & -18.3317 & 51.0181 & 6.2301 & 90 & 3.955 & -22.7034 & 65.6591 & 0.0591 \\
\hline 40 & 2.910 & -18.9594 & 63.6600 & 0.9122 & 91 & 3.967 & -26.2036 & 65.9716 & 3.3246 \\
\hline 41 & 2.985 & -15.3933 & 57.0712 & -1.0020 & 92 & 3.975 & -23.9063 & 67.9993 & 3.2393 \\
\hline 42 & 3.007 & -17.7367 & 66.8141 & -0.1239 & 93 & 3.988 & -19.0371 & 63.7315 & -0.5701 \\
\hline 43 & 3.055 & -18.0569 & 51.1561 & 8.7970 & 94 & 4.013 & -20.8216 & 68.6925 & 1.1507 \\
\hline 44 & 3.084 & -15.3697 & 56.2202 & 1.8015 & 95 & 4.045 & -26.7432 & 38.6059 & 15.6954 \\
\hline 45 & 3.114 & -10.7253 & 49.6106 & -10.9936 & 96 & 4.047 & -20.6688 & 64.3567 & 1.5472 \\
\hline 46 & 3.120 & -21.7260 & 65.8043 & 0.3584 & 97 & 4.057 & -20.5584 & 65.4433 & 0.9534 \\
\hline 47 & 3.130 & -16.4089 & 47.6659 & 12.2466 & 98 & 4.081 & -20.8556 & 66.4925 & 2.5085 \\
\hline 48 & 3.137 & -11.1805 & 50.0712 & -10.5065 & 99 & 4.093 & -22.0741 & 63.3852 & 0.5779 \\
\hline 49 & 3.170 & -17.2828 & 54.0629 & 6.3039 & 100 & 4.098 & -24.2912 & 55.8186 & -0.1082 \\
\hline 50 & 3.187 & -8.4942 & 45.8444 & -8.5054 & & & & & \\
\hline
\end{tabular}


Table S6: Energies $E_{i}[\mathrm{eV}]$ and dipole moments $\boldsymbol{\mu}_{i i} X, Y, Z$ components [D] obtained by ZINDO/S for reduced state on oxidized-state geometry.

\begin{tabular}{|c|c|c|c|c|c|c|c|c|c|}
\hline $\mathrm{i}$ & $E_{i}$ & $\mu_{i i}^{x}$ & $\mu_{i i}^{y}$ & $\mu_{i i}^{z}$ & $\mathrm{i}$ & $E_{i}$ & $\mu_{i i}^{x}$ & $\mu_{i i}^{y}$ & $\mu_{i i}^{z}$ \\
\hline 0 & 0.000 & $\frac{22}{-17.5088}$ & 67.5256 & 3.4415 & 51 & 4.118 & -37.5420 & 51.5434 & -3.7995 \\
\hline 1 & 0.835 & -17.1255 & 67.6112 & 3.1898 & 52 & 4.124 & -15.3095 & 61.9977 & -2.0749 \\
\hline 2 & 0.885 & -17.2589 & 67.5696 & 3.2604 & 53 & 4.161 & -19.9569 & 26.7008 & 9.1816 \\
\hline 3 & 1.116 & -17.4078 & 67.6045 & 3.3766 & 54 & 4.232 & -15.3758 & 26.6148 & -4.3649 \\
\hline 4 & 1.762 & -17.1832 & 66.5575 & 3.0273 & 55 & 4.237 & 0.3490 & 70.2605 & -12.0270 \\
\hline 5 & 1.804 & -17.7179 & 68.1708 & 3.8134 & 56 & 4.261 & -18.4428 & 26.3735 & 4.7190 \\
\hline 6 & 2.069 & -17.3313 & 67.5618 & 3.2427 & 57 & 4.293 & -29.4785 & 24.3409 & -4.6538 \\
\hline 7 & 2.198 & -17.3390 & 67.5973 & 3.3443 & 58 & 4.337 & -17.1725 & 66.5386 & 3.4537 \\
\hline 8 & 2.224 & -17.2547 & 67.6357 & 3.2696 & 59 & 4.340 & -37.0593 & 21.1186 & 19.9333 \\
\hline 9 & 2.598 & -16.1530 & 66.1718 & 4.6644 & 60 & 4.358 & -27.2138 & 59.7435 & 4.4671 \\
\hline 10 & 2.673 & -16.8277 & 66.6142 & 1.5046 & 61 & 4.372 & -19.3842 & 65.8565 & 3.7219 \\
\hline 11 & 2.692 & -16.7009 & 66.8114 & 2.8618 & 62 & 4.383 & -18.8781 & 34.3174 & 0.1597 \\
\hline 12 & 2.889 & -22.6046 & 39.8379 & 2.6471 & 63 & 4.407 & -16.7257 & 65.2082 & 2.6074 \\
\hline 13 & 2.896 & -16.3264 & 65.7997 & 3.9892 & 64 & 4.422 & 1.2257 & 57.5830 & -5.5117 \\
\hline 14 & 2.958 & -16.5318 & 64.3698 & 4.6080 & 65 & 4.454 & -19.0904 & 71.0536 & 3.4780 \\
\hline 15 & 3.017 & -16.9697 & 66.4967 & 2.9681 & 66 & 4.470 & -19.4038 & 62.6315 & 2.7836 \\
\hline 16 & 3.123 & -18.3601 & 67.0863 & 2.7384 & 67 & 4.494 & -16.7872 & 11.7101 & 16.8721 \\
\hline 17 & 3.143 & -14.6391 & 57.5264 & 7.3624 & 68 & 4.514 & -21.9377 & 58.1373 & 1.9419 \\
\hline 18 & 3.176 & -28.9604 & 63.1899 & 5.9467 & 69 & 4.530 & -26.0879 & 59.2128 & 5.0704 \\
\hline 19 & 3.178 & -16.7609 & 65.5753 & 3.3182 & 70 & 4.532 & -18.1221 & 64.8312 & 3.4824 \\
\hline 20 & 3.213 & -15.9048 & 65.6137 & 3.0201 & 71 & 4.547 & -16.8468 & 11.2159 & 16.9250 \\
\hline 21 & 3.222 & -17.7001 & 68.9280 & 3.6766 & 72 & 4.550 & -17.2577 & 61.5185 & 3.1313 \\
\hline 22 & 3.233 & -17.0958 & 66.1072 & 3.7040 & 73 & 4.550 & -18.8302 & 54.8397 & 0.7085 \\
\hline 23 & 3.274 & -10.9021 & 54.7662 & -3.9481 & 74 & 4.567 & -37.5224 & 50.3882 & -2.6908 \\
\hline 24 & 3.347 & -13.1883 & 47.0993 & 12.5967 & 75 & 4.612 & -10.4365 & 15.9644 & -11.2709 \\
\hline 25 & 3.364 & -41.6857 & 45.1724 & 8.1400 & 76 & 4.621 & -10.3983 & 16.2691 & -11.4169 \\
\hline 26 & 3.381 & -22.5094 & 40.4048 & 2.9287 & 77 & 4.625 & -35.7159 & 18.4911 & 21.5281 \\
\hline 27 & 3.458 & -6.5406 & 48.4785 & -7.8629 & 78 & 4.634 & -17.2769 & 66.0950 & 2.8507 \\
\hline 28 & 3.494 & -28.6365 & 62.9811 & 6.0373 & 79 & 4.638 & -27.3939 & 20.5576 & -6.2909 \\
\hline 29 & 3.532 & -12.5784 & 48.0743 & 14.0842 & 80 & 4.649 & -17.5586 & 66.6263 & 3.5247 \\
\hline 30 & 3.609 & -17.8743 & 68.3190 & 3.1559 & 81 & 4.651 & -28.2153 & 21.4054 & -6.1429 \\
\hline 31 & 3.653 & -12.4354 & 40.6691 & 16.3061 & 82 & 4.667 & -32.4726 & 45.7822 & 9.4545 \\
\hline 32 & 3.662 & -12.6582 & 56.3119 & -4.1391 & 83 & 4.674 & -23.6145 & 48.0114 & 12.5396 \\
\hline 33 & 3.714 & -12.4413 & 40.4150 & 16.7077 & 84 & 4.681 & -34.6809 & 25.9701 & 18.2099 \\
\hline 34 & 3.715 & -4.9535 & 44.2562 & -10.0972 & 85 & 4.688 & 5.0708 & 48.6024 & -1.3938 \\
\hline 35 & 3.717 & -27.0448 & 62.4295 & 5.5982 & 86 & 4.699 & -18.1748 & 64.4462 & 3.4938 \\
\hline 36 & 3.737 & -12.0728 & 42.1465 & 15.7027 & 87 & 4.703 & -35.7651 & 45.6871 & 8.0713 \\
\hline 37 & 3.741 & -5.3598 & 45.7104 & -9.4086 & 88 & 4.717 & -17.2649 & 64.5290 & 3.9665 \\
\hline 38 & 3.752 & -20.0645 & 64.4852 & 4.8025 & 89 & 4.740 & 4.9641 & 47.8871 & -1.3680 \\
\hline 39 & 3.753 & -22.1039 & 63.7544 & 5.2785 & 90 & 4.752 & -15.7762 & 63.0869 & 2.6887 \\
\hline 40 & 3.794 & -11.8256 & 40.2893 & 15.9282 & 91 & 4.783 & -16.0723 & 65.3049 & 2.1538 \\
\hline 41 & 3.818 & -41.4050 & 44.3845 & 8.3050 & 92 & 4.828 & -14.8615 & 60.7047 & 5.6155 \\
\hline 42 & 3.859 & -1.0162 & 76.3989 & -15.8797 & 93 & 4.832 & -21.8722 & 40.2764 & 2.6595 \\
\hline 43 & 3.866 & -3.9428 & 43.3994 & -10.0807 & 94 & 4.846 & -32.2878 & 53.9625 & 5.8364 \\
\hline 44 & 3.872 & -4.9265 & 44.8912 & -9.1295 & 95 & 4.902 & -15.1868 & 61.5358 & 4.1090 \\
\hline 45 & 3.884 & -14.5648 & 58.7291 & 6.1921 & 96 & 4.938 & -14.1975 & 53.8681 & 9.5657 \\
\hline 46 & 3.910 & -15.2970 & 60.6712 & 6.9777 & 97 & 4.946 & -15.8219 & 38.3655 & -8.3786 \\
\hline 47 & 3.968 & -17.3999 & 66.9871 & 3.2776 & 98 & 4.960 & -16.4450 & 38.7119 & -8.5404 \\
\hline 48 & 3.998 & -29.7425 & 58.9612 & 4.0130 & 99 & 4.980 & -26.7354 & 56.1822 & 5.3613 \\
\hline 49 & 4.048 & -17.5090 & 67.9291 & 2.3361 & 100 & 5.011 & -14.2842 & 60.1418 & 2.5154 \\
\hline 50 & 4.099 & -28.0471 & 63.3223 & 5.7958 & & & & & \\
\hline
\end{tabular}




\section{References for Supporting Information}

[1] X. Jiang, Z. Futera, and J. Blumberger. Ergodicity-breaking in thermal biological electron transfer? cytochrome c revisited. J. Phys. Chem. B, 123:7588-7598, 2019.

[2] M. Dinpajooh, D. R. Martin, and D. V. Matyushov. Polarizability of the active site of cytochrome c reduces the activation barrier for electron transfer. Sci. Rep., 6:28152, 2016.

[3] D. R. Martin, M. Dinpajooh, and D. V. Matyushov. Polarizability of the active site in enzymatic catalysis: Cytochrome c. J. Phys. Chem. B, 123(50):10691, 2019. 